\title{
Barrierefreiheit für Gehörlose
}

\section{Gebärdensprachendolmetscher per Liveschaltung auf mobilen Endgeräten}

Sprachbarrieren führen oft zur Fehlinformation für beide Seiten. Bei Krankheit oder Unfällen erschwert dies die Diagnose und schnelle optimale Behandlung. Mit einem Gebärdensprachendolmetscher über mobile Endgeräte soll Gehörlosen und hochgradig Schwerhörigen in Österreich eine flächendeckende, kommunikative Barrierefreiheit in der Gesundheitsversorgung ermöglicht werden.

„Verstehen und verstanden werden ist ein Patientenrecht", stellt Patientenanwalt Dr. Gerald Bachinger fest. Gerade in Ausnahmesituationen, wie es viele Situationen im medizinischen Bereich sind, ergeben sich in der Kommunikation jedoch beträchtliche Hürden für ein gegenseitiges Verstehen. Für Menschen, die in ihrer verbalen Ausdrucksmöglichkeit und ihrer akustischen Wahrnehmung eingeschränkt sind, erhöhen sich diese Hürden zusätzlich. Mit einem neuen Angebot der Plattform Patientensicherheit in Kooperation mit einigen Partnern soll ein Dolmetscher in Gebärdensprachen am Display eines mobilen Endgeräts die Kommunikation verbessern: Bei Behördengängen, Bankterminen und natürlich im Gesundheitsbereich. Ein Pilotprojekt testet seit Anfang Oktober das neue Service.

\section{Ein Drittel des Bedarfs an Gehörlosendolmetschen fehlt}

Der Bedarf an Gebärdendolmetschern ist groß: etwa 8000 Personen in Österreich sind gehörlos, 10.000 bis 15.000 Personen sind hochgradig schwerhörig. Darüber hinaus gibt es eine nicht erfasste Anzahl von Menschen, die aufgrund unterschiedlicher Ursachen in ihrer sprachlichen Kommunikation eingeschränkt sind und sich mit Gebärdensprache verständigen. Die Zahl der Gebärdendolmetscher in Österreich ist jedoch gering, besonders schlecht ist die Versorgung in ländlichen Gebieten. „Etwa ein Drittel des Dolmetscherbedarfs im Bereich der Gebärdensprache kann derzeit in Österreich nicht erfüllt werden“, erklärte Dr. Maria Kletecka-Pulker von der Plattform Patientensicherheit bei der Präsentation des Pilotprojekts Ende September in Wien. Mit dem „Gebärdensprachdolmetscher am

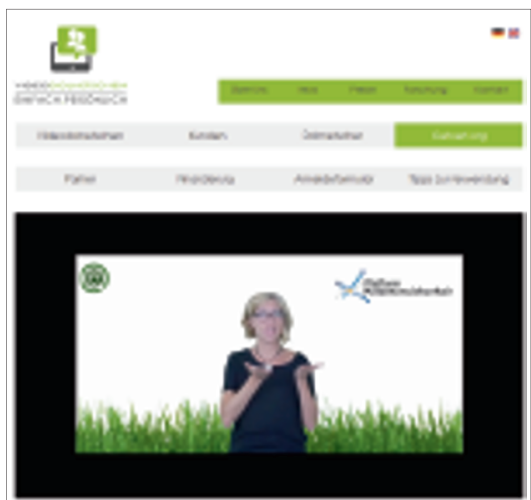

Display" stehen nun 300 Dolmetscher zunächst für die deutsche und österreichische Gebärdensprache von Montag bis Freitag bereit, um per Smartphone, Tablet oder Laptop bei der Verständigung zwischen Gehörlosen und Hörenden unkompliziert und direkt zu unterstützen. Einzige Systemvoraussetzung ist ein LTE/4 G-fähiges Endgerät und ein Mobilfunkstandard von optimal 4G. $k i$

Quelle: www.gehoert.at

\section{wissen \\ ist Macht!} MSc Pflegemanagement

Beginn: 15. März 2016

Dauer: 6 Semester berufsbegleitend, 4 Semester Vollzeit Gebühr: EUR 9.600,-

Abschluss: Master of Science (MSc)

Kontakt: sophie.schwed@donau-uni.ac.at, Tel. +43 (0)2732 893-2745

\section{Wundmanagement}

Beginn: 29. Februar 2016

Dauer: 1 Semester/2 Semester berufsbegleitend

Gebühr: EUR 2.850,-/4.100,-

Abschluss: Zertifikat/Akademische/r Wundmanager/in

Kontakt: christiane.gfoehler@donau-uni.ac.at, Tel. +43 (0)2732 893-2746 